That 100 per cent of bolters were never found was due to a small attack of Botrytis cinerea. The difference in appearance of the four sets of plants was most striking on July 30 . On this date the majority of $V$ plants were $45-60 \mathrm{~cm}$. in height, and the $V X$ plants 15-18 cm., while a few $S$ and $S X$ plants were just showing only the first signs of 'bolting'. Actual flowering dates were for the $V$ plants Aug. 28, VX plants Sept. 2 and $S$ and $S X$ plants Oct. 1. The vernalization treatment of the swollen seed accelerated flowering by thirty-one days. This great acceleration was probably due in part to unfavourable weather during September, retarding the flowering of the $S$ and $S X$ plants. The chief point of interest is that the vernalization treatment given after radicle emergence had occurred was so much less effective than the treatment commenced after the seed had swollen for only twenty-four hours and before germination had begun.

A rather severe attack of Botrytis on a similar series of Webb's Wonderful lettuce was not sufficient to mask similar trends with this variety.

These observations serve to emphasize the importance of the timing of any vernalization treatments. They may not be without their practical significance. Early sowings of a number of crop plants (for example, beet, turnip, celery $y^{3,4}$, etc.) are especially prone to flower prematurely, and this may be due in some cases to a vernalizing effect on the swollen or germinated seed, or on the seedlings, of naturally experienced low soil or atmospheric temperatures in early spring. In so far as the effect is one on the swollen seed and not on the young seedling, the possibility of avoiding these undesirable effects by the sowing of already germinated seed is opened up.

Botany Department,

University, Manchester.

$$
\text { Nov. } 28 .
$$

${ }^{1}$ Grav, S. G., .I. Coun. Sci. Ind. Res. Aust., 15, (3) (1942).

2 Simpson, A. C., Nature, 151 (1943).

"Thompson, H. C. "Premature Seeding of Celery", N.Y. (Cornell) Exp. Sta. Bull. 480 .

‘ Sakr, E. S. M., Proc. Amer. Soc. Hort. Sci., 44 (1944).

\section{Prevention of Oil Shock in the Orchard}

THe use of a highly refined petroleum oil for application to orchard trees as an insecticide and acaricide is firmly established as a valuable pestcontrol treatment with eitrus, but its use on deciduous trees in summer is limited by the fact that, under certain ill-defined conditions, severe leaf and fruitdrop has been known to occur following the use of a 'summer' petroleum spray.

During the seasons 1944, 1945, 1946, we have carried out field experiments in commercial apple orchards designed to determine whether the addition of the plant-growth regulator alpha naphthyl acetic acid to a white-oil spray would prevent the leaf- and fruit-drop which might otherwise be induced. Until this season we failed to produce leaf- or fruit-drop by the application of oil in trials in Kent, Sussex and Essex ; but this year, on a farm in Essex, severe drop of both leaves and apples did occur in the case of the variety Cox's Orange Pippin after spraying with a white-oil emulsion used at 1 per cent $(0.75$ per cent actual oil). The base oil conformed to the official specification for horticultural white oils for orchard use. On adjoining plots alpha naphthyl acetic acid was added to the diluted oil emulsion as sprayed, so that the concentration of the plant-growth regulating substance was 10 parts per million. The spraying was carried out on June 19, 1946, the variety being Cox's Orange Pippin with Worcester Pearmain as pollinator. On August 28, 1946, a count of the fruits remaining on the Cox trees was made, and the average fruits per tree were as follows:

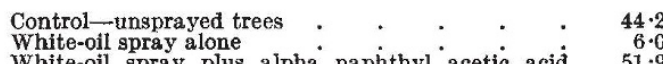

In this case, therefore, the alpha naphthyl acetic acid, a material which is commonly used for the prevention of pre-harvest drop of apples and pears, applied simultaneously with an oil spray, has modified or prevented the shock due to the application of oil and thereby prevented leaf- and fruit-drop.

We regard this result as important since a summer application of white oil to foliage is probably the best method of controlling the serious pest red spider mite (Oligonychus ulmi C. L. Koch) on apples, and hitherto oil has not been widely used for this purpose in Great Britain because of the risk of causing leafand fruit-drop.

\section{W. L. Cole}

W. HeEley

Shell Petroleum Co.

Technical Products Department,

St. Helen's Court, E.C.3. Nov. 21.

\section{Gaseous Sulphur Dioxide and Sodium Bisulphite as Preservatives of Citrus Concentrates}

DIFFERENTIAL preservative efficiency between gaseous sulphur dioxide and sodium bisulphite, observed in citrus concentrates, became of practical significance during the War due to the difficulties in obtaining an adequate supply of gaseous sulphur dioxide in Palestine. Investigating the effect of acidity on the efficiency of various preservatives, Rahn and Conn ${ }^{1}$ found that yeast is checked only by molecular $\mathrm{H}_{2} \mathrm{SO}_{3}$, and not by dissociated ions $\mathrm{HSO}_{3}{ }^{\prime}$ or $\mathrm{SO}_{3}{ }^{\prime \prime}$. According to Downer ${ }^{2}$, quantities ranging between 2,000 and 2,500 p.p.m. sulphur dioxide are necessary for citrus juice concentrates (4:1), and even more are required for higher concentrations, in order to prevent fermentation. This has been explained by him as due to the fact that part of the sulphur dioxide combines with the aldehyde group of the sugar contained in the juice, leaving only part free to act as preservative.

In our experiments, increasing amounts of sodium bisulphite and parallel gaseous sulphur dioxide have been added to concentrated citrus juice $(6: 1)$, inoculated with a known number of wine yeast. The test mixtures, which have been examined periodically during three months, showed the bisulphite to be at least 30-40 per cent more efficient than sulphur dioxide.

The minimum quantity of sodium bisulphite to be added to the concentrate, in order to prevent ferm. entation, has been found by us to range between 1,000 and 1,200 p.p.m., equivalent to $600-700$ p.p.m. sulphur dioxide, while $800-1,000$ p.p.m. gaseous sulphur dioxide, equivalent to $1,300-1,600$ p.p.m. bisulphite, are necessary to produce the same effect.

No significant difference has been observed in the $p H$ of citrus juice concentrate containing either of the two preservatives. Both of them also show the same vitamin $\mathrm{C}$ content. 